\title{
Immune checkpoint inhibitors in small cell lung cancer
}

\author{
Suchita Pakkala, Taofeek K. Owonikoko \\ Department of Hematology and Medical Oncology, Winship Cancer Institute of Emory University, Atlanta, GA, USA \\ Contributions: (I) Conception and design: All authors; (II) Administrative support: None; (III) Provision of study materials or patients: None; (IV) \\ Collection and assembly of data: All authors; (V) Data analysis and interpretation: All authors; (VI) Manuscript writing: All authors; (VII) Final \\ approval of manuscript: All authors. \\ Correspondence to: Taofeek K. Owonikoko, MD, PhD. Department of Hematology and Medical Oncology, Winship Cancer Institute of Emory \\ University, 1365 Clifton Road, NE, Atlanta, GA 30322, USA. Email: towonik@emory.edu.
}

\begin{abstract}
Small cell lung cancer (SCLC) is a rapidly progressive cancer that often debilitates patients within months of detection and quickly becomes refractory to the limited options of therapy. While SCLC is not generally considered an immunogenic tumor, clinical experience suggests that patients with robust immune response manifesting as paraneoplastic syndrome are more likely to present with limited stage of the disease and tend to have a better prognosis. Monoclonal antibodies targeting critical negative regulators of immune response, so called immune checkpoints, such as cytotoxic T-lymphocyte-associated protein 4 (CTLA-4) and programmed death 1 (PD-1) have expanded the application of immune-based therapies to increasing number of advanced stage cancers. These agents overcome the inhibitory immune signals leading to a heightened immune response against cancer cells. These immune checkpoint inhibitors have established efficacy leading to regulatory approval for their use in many cancer types including non-small cell lung cancer (NSCLC). Evaluation of the CTLA-4 inhibitor, ipilimumab and PD-1 inhibitors, nivolumab and pembrolizumab in SCLC have shown encouraging signal but definitive studies are still ongoing. In this review, we discuss the rationale behind the use of checkpoint inhibitors in SCLC, contextualize the results of early trials of immunotherapy agents in SCLC and project the future evolution of this strategy.
\end{abstract}

Keywords: Small cell lung cancer (SCLC); immunotherapy; immune checkpoint; programmed cell death protein 1 (PD-1); programmed death-ligand 1 (PD-L1)

Submitted Sep 26, 2017. Accepted for publication Dec 04, 2017.

doi: $10.21037 /$ jtd.2017.12.51

View this article at: http://dx.doi.org/10.21037/jtd.2017.12.51

\section{Introduction}

Patients with small cell lung cancer (SCLC) have a dismal prognosis. The majority of SCLC patients $(\sim 60 \%)$ are diagnosed with the extensive stage (ES) of the disease and have a median overall survival (OS) of approximately 10 months when treated with standard frontline platinumbased doublet chemotherapy (1-4). In patients with a partial response, consolidative thoracic radiation may improve survival, but 2 year survival for these patients remains poor at $\sim 10 \%(5,6)$. Additionally, more than a third of all newly diagnosed SCLC patients in the US are never treated, often times because of significant debilitation that preclude them from undergoing active treatment $(7,8)$. The outcome for patients diagnosed with limited-stage disease (LD) is only marginally better with a median OS of approximately 2 years when treated with platinum-based chemotherapy and concurrent radiation (9-11). Treatment options for patients with progressive disease beyond the frontline are generally ineffective and associated with significant toxicities. While prophylactic cranial irradiation (PCI) further improves survival for limited stage (LS)-SCLC by $5.4 \%$ at 3 years especially in patients who achieve objective response to concurrent therapy, only $20 \%$ of patients are alive at 3 years (12). Survival benefit of PCI in patients with extensive stage disease is unclear despite clear reduction in the incidence of brain metastasis in two prospective randomized studies in 
this population $(13,14)$. Patients with small primary tumors (T1 or T2) without nodal involvement do particularly well with complete surgical resection followed by adjuvant chemotherapy with a 5 -year survival of only about $50 \%$ (15). However, this is a very rare subset of SCLC patients. Therefore, systemic therapy still remains the bedrock of treatment for the vast majority of SCLC patients.

Due to the highly proliferative nature of SCLC, there is a robust response of the disease to the initial chemotherapy. However, there is a near complete resistance to subsequent chemotherapy following disease progression (16). Response rates to second-line topotecan are $20-25 \%$ with 1-year survivals of $10-30 \%$ (16-18). Ultimately, treatment toxicity coupled with the underlying disease itself can whittle away at a patient's remaining life. A multitude of clinical trials conducted over the past 2 decades have failed to establish new treatment options. A review of patient outcomes over a 2-decade time span showed only modest survival impact of systemic chemotherapy in the real-world management of SCLC patients (8).

Immunotherapy offers a new hope to patients with SCLC. While cancer directed therapies have tried to target mechanisms of growth exploited by these cells, it often impacts normal cells resulting in significant and often intolerable toxicities. Eventually, cancer cells adapt using alternative pathways to develop resistance to those therapies. Additionally, pre-existing heterogeneity within a tumor may mean that only cancer cells susceptible to those therapies respond leaving the resistant cells behind to proliferate. Hence, the interest in harnessing the body's defense system to regulate its own aberrant growth.

\section{Immune surveillance and tumor development}

Physiologic and pathologic cellular changes leading to altered self is recognized by the body's immune mechanism. As a consequence of neoplastic transformation, tumor associated antigens can be displayed via major histocompatibility complex (MHC) I on antigen presenting cells (APCs) to the T cell receptor (TCR) on CD8 cytotoxic $\mathrm{T}$ cells. While all cells express MHC I, T cell activation following antigen recognition in the context of MHC I antigen requires a secondary co-stimulatory signal that occurs with the binding of APC B7 to CD28 on cytotoxic $\mathrm{T}$ cells, leading to immunological elimination of such altered cells. Contrarily, increased expression of cytotoxic T-lymphocyte-associated protein 4 (CTLA-4) is a negative inhibitory feedback on activated $\mathrm{T}$ cells following the binding of B7 with a higher affinity than CD28 (19).
Regulatory T cells (Tregs) constitutively express CTLA-4 and further restrain the activated immune response ultimately suppressing auto-reactivity. Similar to CTLA-4, programmed cell death protein 1 (PD-1) expression on activated $\mathrm{T}$ cells, peripheral $\mathrm{B}$ cells, and myeloid cells also act as inhibitory immune checkpoints. However, in contrast to CTLA-4, binding to its ligand (PD-L1) on tissue or hematopoietic cells is a late phase effect that suppresses activated $T$ cells mainly in the peripheral immune effector sites rather than in the central lymphoid organ sites $(20,21)$.

The expression of tumor specific antigens on cancer cells facilitates recognition by host immune T-cells. Tumor establishment and progression therefore requires evasion of the body's immune surveillance mechanism (22). CTLA-4 and PD-1 are well-established and characterized inhibitory pathways that contribute to a cancer's ability to evade immune surveillance. Checkpoint inhibition of CTLA-4 and PD-1 has proven successful in controlling cancer progression as evidenced by controlled studies in various tumor types leading to regulatory approval of immune checkpoint inhibitors for the treatment of melanoma, NSCLC, head and neck cancers, renal cell cancer, bladder cancers, and microsatellite instability high advanced solid tumors. Post hoc correlative analysis showed that objective tumor responses to immune checkpoint inhibitors occur in cancers with a high mutational burden and antigen load. SCLC has one of the highest rates of mutation burden suggesting that this disease will be particularly susceptible to immune-based therapeutic approaches (23-28). Further support for this therapeutic strategy came from prior findings of the association between heightened immune response and improved SCLC outcomes. Also, high levels of tumor infiltrating immune cells are associated with improved overall survival irrespective of tumor stage, patient performance status, or type of treatment received (29). More specifically, a high ratio of $\mathrm{T}$ effector (Teff) to Tregs, known to inhibit anti-tumor responses in SCLC, has been associated with better survival while those with recurrent disease have a low ratio $(30,31)$. Additionally, the development of immune mediated paraneoplastic syndromes such as LambertEaton myasthenia syndrome (LEMS) as a result of cross reactivity of antibodies against tumor specific antigens on SCLC with neuronal tissue appears to be associated with a favorable outcome compared to those without paraneoplastic syndrome $(32,33)$. Moreover, SCLC patients with LEMS showed a reduced level of suppressive Treg lymphocyte subset in the peripheral lymphocyte compartment supporting the notion that paraneoplastic syndrome will be accompanied 
Table 1 Completed immunotherapy trials in ES-SCLC

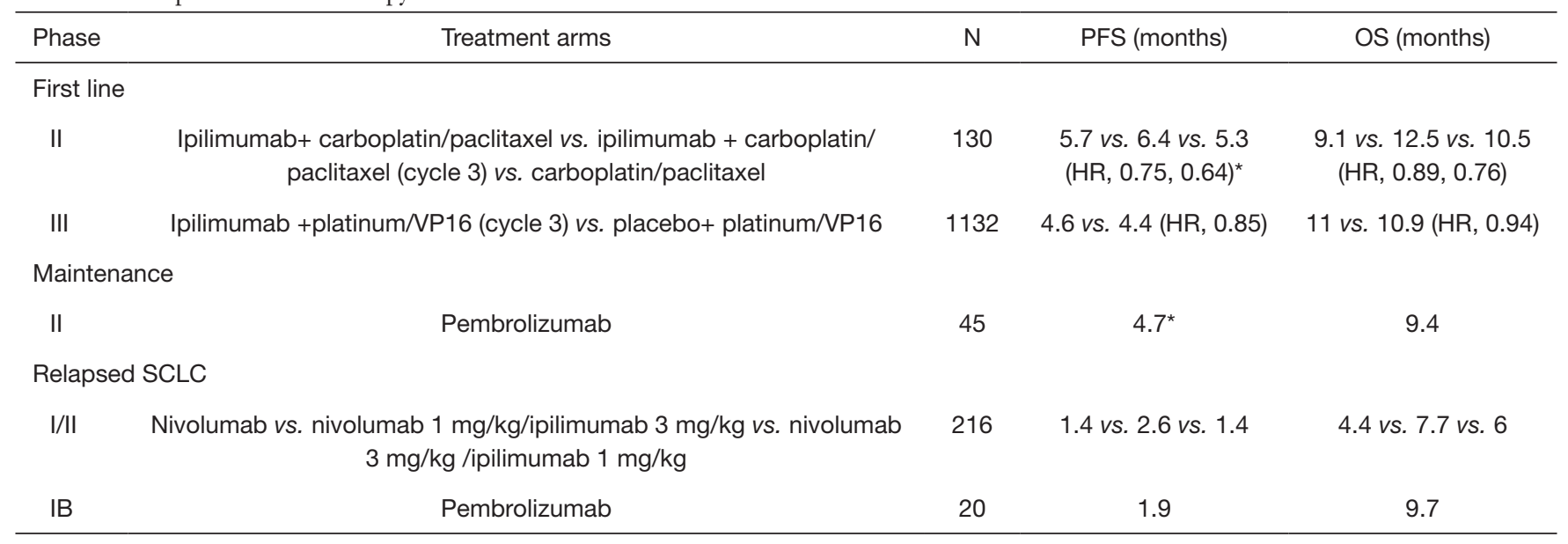

*, irPFS. ES-SCLC, extensive stage small cell lung cancer; PFS, progression free survival; OS, overall survival; N, number; VP16, etoposide; HR, hazard ratio.

by potent antitumor immunity (34). Therefore, therapeutic enhancement of immunotherapy is a reasonable approach and is currently an active area of clinical research to establish novel treatment options for SCLC patients.

\section{CTLA-4 inhibition}

Ipilimumab, an anti-CTLA-4 monoclonal antibody was the first immune checkpoint agent prospectively studied in SCLC where it was combined with chemotherapy in ES-SCLC. A 3-arm phase 2 trial in treatment naïve ESSCLC patients compared carboplatin/paclitaxel doublet alone with ipilimumab administered concurrently with carboplatin/paclitaxel for 2 cycles followed by chemotherapy and phased combination of ipilimumab starting with cycle 3 of chemotherapy. The immune related PFS (irPFS) was significantly prolonged for the phased ipilimumab arm (5.7 vs. 6.4 vs. 5.3 months; HR: 0.75, P=0.11 for concurrent and HR: 0.64, $\mathrm{P}=0.03$ for phased compared to control) (Table 1) (35). There was also a non-significant trend in OS (10.5 vs. $9.1 \mathrm{vs}$. 12.5 months; HR, 0.89; CI, 0.57-1.39; 0.76, CI, 0.48-1.19 compared to control) that favored the phased ipilimumab arm. The efficacy signal was also accompanied by increased toxicity whereby grade $3 / 4$ toxicities were more common in the ipilimumab arms (30\% control vs. $43 \%$ concurrent vs. $50 \%$ phased). However, the rates of treatment discontinuation due to toxicity were similar. A confirmatory phase III evaluation of ipilimumab in combination with chemotherapy starting with cycle 3 of platinum/etoposide with maintenance ipilimumab given every 12 weeks failed to improve OS over chemotherapy alone with median OS of 11 vs. 10.9 months; HR, 0.94; CI, 0.81-1.09) (36). There was also no significant improvement in median progression free survival (4.6 vs. 4.4 months; HR, 0.85; CI, 0.75-0.97). Overall response rate (ORR) was identical at $62 \%$ in each arm and the duration of response was also comparable at $4.01 \mathrm{vs} .3 .45$ months for the ipilimumab and chemotherapy alone arms, respectively. Immune-related toxicities including diarrhea, rash and colitis lead to higher rates of treatment discontinuation ( $18 \%$ vs. $2 \%$ ), and deaths ( $5 \%$ vs. $2 \%$ ). The reason for the failure of the confirmatory phase III trial to replicate the signal observed in the initial phase II trial of chemotherapy plus ipilimumab is not clear. However, various factors have been suggested including the different chemotherapy backbone employed in the phase II trial (carboplatin/paclitaxel) and the phase III trial (carboplatin/ etoposide). Potential differences in the patient population enrolled could have also contributed since both trials did not employ any predictive biomarker to enrich for patients likely to benefit from chemoimmunotherapy. In addition, the initial signal of efficacy in the phase II study was based on a novel endpoint of irPFS, which had not been validated as a reliable surrogate for OS in this patient population. Most importantly, ipilimumab has limited tumor specific immune modulatory effect since CTLA4 signaling is more relevant in the relatively nonspecific activation phase of the immune response (37). Perhaps, chemoimmunotherapy combination targeting the immune effector phase with antiPD-1 antibodies would be expected to yield better result. 


\section{Targeting PD-1}

Relapsed SCLC: The initial evaluation of immune checkpoint inhibition strategy in SCLC using ipilimumab, in combination with chemotherapy was ineffective in the frontline setting. However, the strategy targeting PD-1 pathway alone or in combination with anti-CTLA4 agents has met with better success in previously treated patients. The phase I/II CheckMate 032 trial (Table 1) explored the efficacy of nivolumab alone or in combination with ipilimumab in patients who have progressed following prior systemic chemotherapy (38). Eligible patients were required to have failed at least one line of a platinum-containing systemic therapy were assigned to nivolumab monotherapy ( $3 \mathrm{mg} / \mathrm{kg}$ ) or nivolumab plus ipilimumab $(1 \mathrm{mg} / \mathrm{kg}$ plus $1 \mathrm{mg} / \mathrm{kg} ; 1 \mathrm{mg} / \mathrm{kg}$ plus $3 \mathrm{mg} / \mathrm{kg}$ or $3 \mathrm{mg} / \mathrm{kg}$ plus $1 \mathrm{mg} / \mathrm{kg}$ ) followed by nivolumab maintenance therapy. Ten of the 98 patients in the monotherapy arm responded (10\% ORR) while the combination doses appeared to have a higher ORRs at $33 \%$ ( 1 of 3 patients), $23 \%$ (14 of 61 patients), and $19 \%$ (10 of 54 patients), respectively. Responses were seen regardless of the number of prior therapy and platinum sensitivity. One-year OS for this patient population in whom the majority had 2 or more lines of therapy was $33 \%$ for nivolumab, $43 \%$ with the $1 \mathrm{mg} / \mathrm{kg}$ plus $3 \mathrm{mg} / \mathrm{kg}$ dose, and $35 \%$ with the $3 \mathrm{mg} / \mathrm{kg}$ plus $1 \mathrm{mg} / \mathrm{kg}$ dose. The most common severe side effects observed with these regimens were elevated lipase and diarrhea, but treatment discontinuation was less than $12 \%$ and comparable among the arms. However, the combination arms with higher doses of ipilimumab had a higher frequency of grade 3 or 4 adverse events at $30 \%$ and $19 \%$, respectively vs. $13 \%$ with nivolumab alone. An updated result of the Checkmate 032 study was presented at the 2017 ASCO annual meeting including data from the randomized cohorts added to the initial non-randomized cohorts (39). The more robust data from approximately 400 patients enrolled on the study confirmed the initial efficacy signal with a doubling of the response rate in patients treated with the combination versus nivolumab alone ( $22 \%$ vs. $9 \%)$. Three treatment related deaths occurred in the higher dose combination arms and were due to myasthenia gravis, acute renal failure, and pneumonitis. The overall positive benefit risk balance of this treatment strategy especially, the durable response and OS signal informed the adoption of this regimen by the NCCN guideline committee as a reasonable option in previously treated SCLC patients. A definitive randomized phase III trial, Checkmate
331 comparing topotecan to nivolumab as second line treatment for SCLC has completed enrolment and results are awaited. Pembrolizumab, another monoclonal antibody against PD-1 was tested in the phase I KEYNOTE 028 study. This PD-1 antibody similarly demonstrated a promising response rate of $33 \%$ (8/24 patients) with median duration of response of 19.4 months and 1-year survival of $37.7 \%$ in previously treated PD-L1 positive ( $\geq 1 \%$ staining) ES-SCLC patients (40). The vast majority (87.5\%) of the patients were previously treated with 2 or more lines of therapy. Sixty-seven percent of patients had an AE with the most common being fatigue, rash, diarrhea, and arthralgia. However, only $8.3 \%$ had a grade 3 or higher event while $12.5 \%$ had an immune mediated $\mathrm{AE}$ such as thyroiditis or colitis.

Maintenance immunotherapy: There is no established agent employed as maintenance therapy following frontline chemotherapy for SCLC. However, temsirolimus and sunitinib both showed signal of benefit in small phase II studies $(41,42)$. Immune checkpoint inhibitors are also being evaluated as maintenance therapy in SCLC. A multicenter phase II trial of maintenance pembrolizumab in 45 patients showed no improvement in PFS (1.4, CI, 1.3-4) months when patients received pembrolizumab after completing 4-6 cycles of platinum/etoposide (43). Exploratory analysis showed a promising signal in terms of irPFS with a median of 4.7 (CI, 1.8-6.7) months and especially in patients with $\mathrm{PD}-\mathrm{L} 1$ positive tumors compared to PD-L1 negative patients (5.5 vs. 1.3 months). The median OS was 9.4 months (CI, 6.1-15.2) and $30 \%$ at 1 year. Common AEs were as fatigue, nausea, and dyspnea. Nivolumab and the combination of nivolumab and ipilimumab are currently being evaluated as maintenance therapy after standard therapy in limited stage SCLC (STIMULI trial) and extensive stage SCLC (CheckMate 451 trial).

Immunotherapy in the frontline: PD-1 antibodies are under active investigation as part of the frontline regimen for extensive stage SCLC trials (Table 2). REACTION is a phase II trial evaluating platinum/etoposide in treatment naïve patients with or without pembrolizumab while KEYNOTE 604 is a definitive phase III trial of the same chemoimmunotherapy regimen in comparison to standard chemotherapy. Ongoing studies in previously treated ES-SCLC patients include: pembrolizumab vs. topotecan (AFT-17) and nivolumab vs. topotecan or amrubicin (CheckMate 331). PD-L1 binds B71 with 
Table 2 Ongoing immunotherapy studies in SCLC

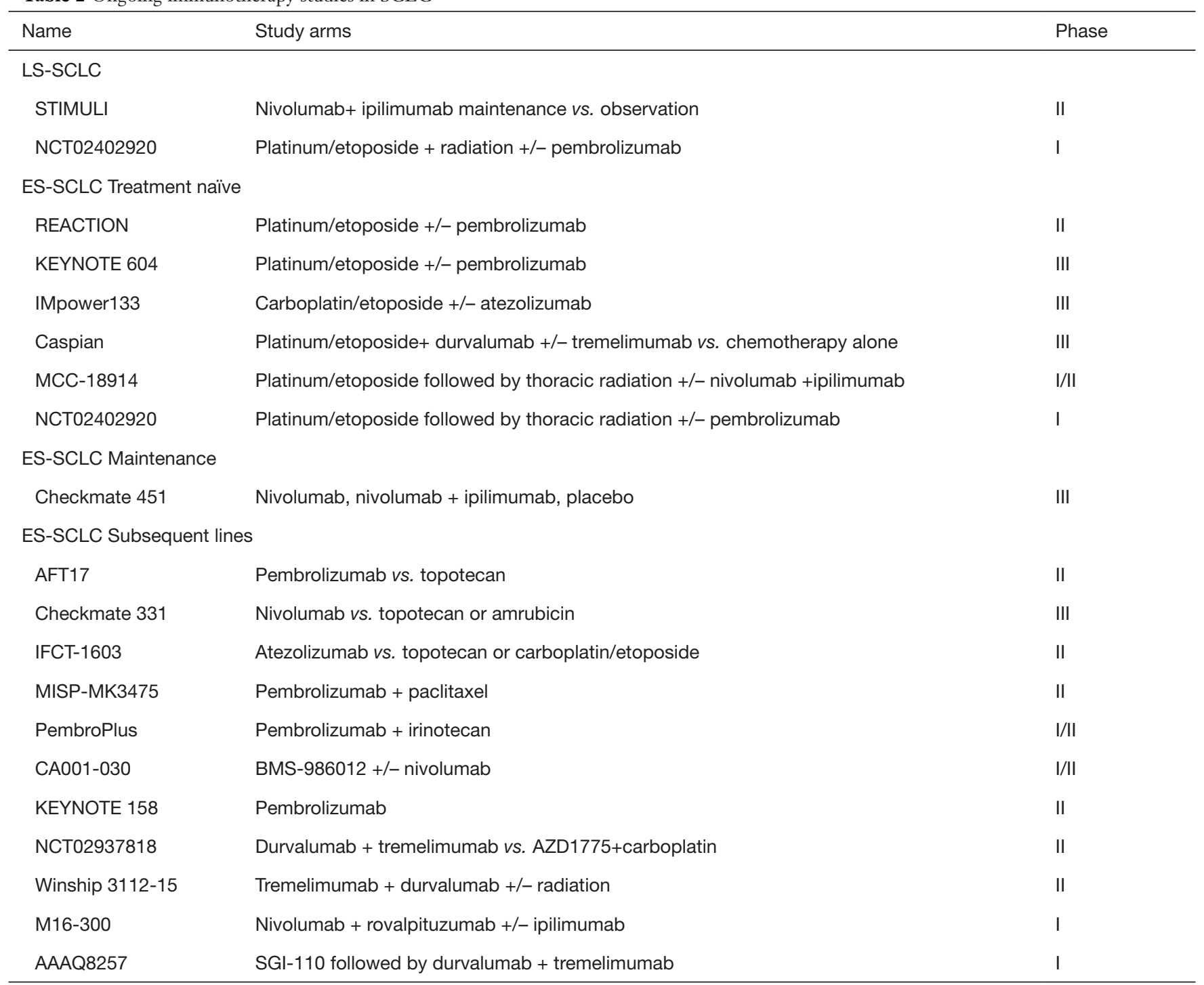

ES-SCLC, extensive stage small cell lung cancer; LS, limited stage.

greater affinity than CD28 and may therefore inactivate T-cells through a PD-1 independent mechanism. Thus, ligand targeting PD-L1 inhibitors such as atezolizumab and durvalumab may have efficacy advantage over inhibitors that bind to PD-1 receptors such as nivolumab and pembrolizumab at least theoretically in the absence of clinical comparison (44). Hence, atezolizumab is being studied in combination with platinum/etoposide and compared to second line chemotherapy while durvalumab is being evaluated in combination with another CTLA-4 inhibitor, tremelimumab with first line chemotherapy and in refractory SCLC.

\section{Future approaches}

Another avenue of incorporating immunotherapy is by combining it with radiation. Radiation has shown to be immunogenic and can theoretically prime the cancer to immunotherapy. Radiation causes cellular apoptosis and exposes the immune system to additional antigens (45). MHC I protein which is poorly expressed in SCLC can be induced along with other neoantigens following ionizing radiation treatment $(46,47)$. Radiation can also remodel the tumor microenvironment in part by reducing the amount of mesenchymal-derived suppressor cells so that the tumor 
stroma becomes more conducive for CD8 cytotoxic T cell activation and increased presence of immune effector cells (48-50). In addition to direct local immune promoting effect, radiation can also induce systemic immune response leading to tumor control at sites beyond the immediate radiation field, so called abscopal effect (51). Ionizing radiation is an established component of treatment for limited stage SCLC and new data suggests potential benefit of consolidation radiation therapy in extensive stage SCLC. Therefore, the combination of immunotherapy and radiation is a rational progression of therapeutic strategy for this disease. Indeed, this concept is under exploration in three prospective clinical trials in limited and extensive stage disease: (I) the STIMULI trial of ipilimumab and nivolumab after standard concurrent chemoradiation in LS-SCLC; (II) dose escalated pembrolizumab concurrent with chemoradiation in LS-SCLC or with consolidative thoracic radiation in ES-SCLC followed pembrolizumab maintenance; and (III) Tremelimumab and durvalumab with or without preceding SBRT to assess for the abscopal effect (Table 2).

In addition to attempts to enhance the effects of immunotherapy, there are also efforts underway to better identify those who are likely to respond to therapy. Currently, there is no perfect biomarker that can accurately predict clinical response to immunotherapy. Although a higher proportion of PD-L1 positive NSCLC patients responded to anti-PD-1 therapy and with higher response rates than $\mathrm{PD}-\mathrm{L} 1$ negative patients, durable responses seen in up to $20 \%$ of patients with $<1 \%$ PD-L1 expression (52-55). A pre-planned analysis of PD-L1 status and clinical outcome in the CheckMate 032 study in SCLC patients revealed that responses occurred irrespective of PD-L1 expression (38). Contrarily, exploratory analysis from the maintenance pembrolizumab study showed the pattern of PD-L1 expression at the tumor-stroma interface rather than the degree of expression to be associated with clinical benefit (43). Therefore, the role of PD-L1 expression in SCLC will have to be further defined in ongoing studies along with other biomarkers such as tumor infiltrating immune cells, mutational burden and immune gene signatures currently being investigated to guide immunotherapy.

\section{Conclusions}

Initial checkpoint inhibition trials have demonstrated encouraging results with favorable responses and generally manageable toxicities in SCLC patients with otherwise limited treatment options. While the addition of ipilimumab to chemotherapy yielded some disappointing results in the first line setting, the durable response and prolonged survival observed with nivolumab/ipilimumab or single agent pembrolizumab for refractory SCLC patients is promising. Additional strategies to incorporate immune targeted agents to the management of SCLC range from maintenance therapy with single or double immune checkpoint agents to combination with chemotherapy and radiation. Unlike the failed attempts to date to establish a role for biologically targeted agents in the treatment of SCLC, early results indicate that immunotherapy will have a role in the management of this difficult disease.

\section{Acknowledgements}

None.

\section{Footnote}

Conflicts of Interest: The authors have no conflicts of interest to declare.

\section{References}

1. Govindan R, Page N, Morgensztern D, et al. Changing epidemiology of small-cell lung cancer in the united states over the last 30 years: analysis of the surveillance, epidemiologic, and end results database. J Clin Oncol 2006;24:4539-44.

2. Socinski MA, Smit EF, Lorigan P, et al. Phase III study of pemetrexed plus carboplatin compared with etoposide plus carboplatin in chemotherapy-naive patients with extensive-stage small-cell lung cancer. J Clin Oncol 2009;27:4787-92.

3. Lara PN, Natale R, Crowley J, et al. Phase III Trial of Irinotecan/Cisplatin Compared With Etoposide/Cisplatin in extensive-stage small-cell lung cancer: clinical and pharmacogenomic results from SWOG S0124. J Clin Oncol 2009;27:2530-5.

4. Rossi A, Maio MD, Chiodini P, et al. Carboplatin- or cisplatin-based chemotherapy in first-line treatment of small-cell lung cancer: the COCIS meta-analysis of individual patient data. J Clin Oncol 2012;30:1692-8.

5. Slotman BJ, van Tinteren H, Praag JO, et al. Use of thoracic radiotherapy for extensive stage small-cell lung cancer: a phase 3 randomised controlled trial. Lancet 2015;385:36-42.

6. Slotman BJ, van Tinteren H, Praag JO, et al. Radiotherapy for extensive stage small-cell lung cancer - authors' reply. 
Lancet 2015;385:1292-3.

7. Small AC, Tsao C-K, Moshier EL, et al. Prevalence and characteristics of patients with metastatic cancer who receive no anticancer therapy. Cancer 2012;118:5947-54.

8. Behera M, Ragin C, Kim S, et al. Trends, predictors, and impact of systemic chemotherapy in small cell lung cancer patients between 1985 and 2005. Cancer 2016;122:50-60.

9. Takada M, Fukuoka M, Kawahara M, et al. Phase III study of concurrent versus sequential thoracic radiotherapy in combination with cisplatin and etoposide for limited-stage small-cell lung cancer: results of the japan clinical oncology group study 9104. J Clin Oncol 2002;20:3054-60.

10. Turrisi AT, Kim K, Blum R, et al. Twice-daily compared with once-daily thoracic radiotherapy in limited smallcell lung cancer treated concurrently with cisplatin and etoposide. New Engl J Med 1999;340:265-71.

11. Jänne PA, Freidlin B, Saxman S, et al. Twenty-five years of clinical research for patients with limited-stage small cell lung carcinoma in North America. Cancer 2002;95:1528-38.

12. Aupérin A, Arriagada R, Pignon JP, et al. Prophylactic cranial irradiation for patients with small-cell lung cancer in complete remission. New Engl J Med 1999;341:476-84.

13. Slotman B, Faivre-Finn C, Kramer G, et al. Prophylactic cranial irradiation in extensive small-cell lung cancer. $\mathrm{N}$ Engl J Med 2007;357:664-72.

14. Takahashi T, Yamanaka T, Seto T, et al. Prophylactic cranial irradiation versus observation in patients with extensive-disease small-cell lung cancer: a multicentre, randomised, open-label, phase 3 trial. Lancet Oncol 2017;18:663-71.

15. Yang C-FJ, Chan DY, Speicher PJ, et al. Role of adjuvant therapy in a population-based cohort of patients with early-stage small-cell lung cancer. J Clin Oncol 2016;34:1057-64.

16. Owonikoko T, Behera M, Chen Z, et al. Meta-analysis of second line chemotherapy efficacy in sensitive and refractory small cell lung cancer (SCLC) Patients. J Thorac Oncol 2010;5:S551-2.

17. von Pawel J, Schiller JH, Shepherd FA, et al. Topotecan versus cyclophosphamide, doxorubicin, and vincristine for the treatment of recurrent small-cell lung cancer. J Clin Oncol 1999;17:658-67.

18. Eckardt JR, Pawel Jv, Pujol JL, et al. Phase III study of oral compared with intravenous topotecan as secondline therapy in small-cell lung cancer. J Clin Oncol 2007;25:2086-92.
19. Spigel DR, Socinski MA. Rationale for Chemotherapy, Immunotherapy, and Checkpoint Blockade in SCLC: Beyond Traditional Treatment Approaches. J Thorac Oncol 2013;8:587-98.

20. Iwai Y, Hamanishi J, Chamoto K, et al. Cancer immunotherapies targeting the PD-1 signaling pathway. J Biomed Sci 2017;24:26.

21. Buchbinder EI, Desai A. CTLA-4 and PD-1 Pathways: Similarities, Differences, and Implications of Their Inhibition. Am J Clin Oncol 2016;39:98-106.

22. Gajewski TF, Schreiber H, Fu YX. Innate and adaptive immune cells in the tumor microenvironment. Nat Immunol 2013;14:1014-22.

23. Chalmers ZR, Connelly CF, Fabrizio D, et al. Analysis of 100,000 human cancer genomes reveals the landscape of tumor mutational burden. Genome Med 2017;9:34.

24. Le DT. PD-1 Blockade in tumors with mismatch-repair deficiency. N Engl J Med 2015;372:2509-20.

25. Rizvi NA, Hellmann MD, Snyder A, et al. Cancer immunology. Mutational landscape determines sensitivity to PD-1 blockade in non-small cell lung cancer. Science 2015;348:124-8.

26. Snyder A, Makarov V, Merghoub T, et al. Genetic basis for clinical response to CTLA-4 blockade in melanoma. N Engl J Med 2014;371:2189-99.

27. Rosenberg JE, Hoffman-Censits J, Powles T, et al. Atezolizumab in patients with locally advanced and metastatic urothelial carcinoma who have progressed following treatment with platinum-based chemotherapy: a single-arm, multicentre, phase 2 trial. Lancet 2016;387:1909-20.

28. McGranahan N, Furness AJS, Rosenthal R, et al. Clonal neoantigens elicit $\mathrm{T}$ cell immunoreactivity and sensitivity to immune checkpoint blockade. Science 2016;351:1463-9.

29. Wang W, Hodkinson P, McLaren F, et al. Histologic assessment of tumor-associated CD45+ cell numbers is an independent predictor of prognosis in small cell lung cancer. Chest 2013;143:146-51.

30. Shimizu J, Yamazaki S, Sakaguchi S. Induction of tumor immunity by removing CD25+CD4+ T cells: a common basis between tumor immunity and autoimmunity. J Immunol 1999;163:5211-8.

31. Koyama K, Kagamu H, Miura S, et al. Reciprocal CD4+ T-cell balance of effector CD62Llow CD4+ and CD62LhighCD25+CD4+ regulatory T cells in small cell lung cancer reflects disease stage. Clin Cancer Res 2008;14:6770-9.

32. Maddison P, Newsom-Davis J, Mills KR, et al. Favourable 
prognosis in Lambert-Eaton myasthenic syndrome and small-cell lung carcinoma. Lancet 1999;353:117-8.

33. Manley GT, Smitt PS, Dalmau J, et al. Hu antigens: Reactivity with hu antibodies, tumor expression, and major immunogenic sites. Ann Neurol 1995;38:102-10.

34. Tani T, Tanaka K, Idezuka J, et al. Regulatory T cells in paraneoplastic neurological syndromes. J Neuroimmunol 2008;196:166-9.

35. Reck M, Bondarenko I, Luft A, et al. Ipilimumab in combination with paclitaxel and carboplatin as first-line therapy in extensive-disease-small-cell lung cancer: results from a randomized, double-blind, multicenter phase 2 trial†. Ann Oncol 2013;24:75-83.

36. Reck M, Luft A, Szczesna A, et al. Phase III randomized trial of ipilimumab plus etoposide and platinum versus placebo plus etoposide and platinum in extensive-stage small-cell lung cancer. J Clin Oncol 2016;34:3740-8.

37. Riess JW, Lara PN, Gandara DR. Theory meets practice for immune checkpoint blockade in small-cell lung cancer. J Clin Oncol 2016;34:3717-8.

38. Antonia SJ, López-Martin JA, Bendell J, et al. Nivolumab alone and nivolumab plus ipilimumab in recurrent smallcell lung cancer (CheckMate 032): a multicentre, openlabel, phase 1/2 trial. Lancet Oncol 2016;17:883-95.

39. Hellmann MD, Ott PA, Zugazagoitia J, et al. Nivolumab (nivo) \pm ipilimumab (ipi) in advanced small-cell lung cancer (SCLC): First report of a randomized expansion cohort from CheckMate 032. J Clin Oncol 2017;35:S8503.

40. Ott P, Felip E, Hiret S, et al. OA05.01 Pembrolizumab in patients with extensive-stage small cell lung cancer: Updated survival results from KEYNOTE-028. J Thorac Oncol;12:S259.

41. Ready NE, Pang HH, Gu L, et al. Chemotherapy with or without maintenance sunitinib for untreated extensivestage small-cell lung cancer: a randomized, doubleblind, placebo-controlled phase II Study-CALGB 30504 (Alliance). J Clin Oncol 2015;33:1660-5.

42. Pandya KJ, Dahlberg S, Hidalgo M, et al. A randomized, phase II trial of two dose levels of temsirolimus (CCI779) in patients with extensive-stage small-cell lung cancer who have responding or stable disease after induction chemotherapy: a trial of the Eastern Cooperative Oncology Group (E1500). J Thorac Oncol 2007;2:1036-41.

Cite this article as: Pakkala S, Owonikoko TK. Immune checkpoint inhibitors in small cell lung cancer. J Thorac Dis 2018;10(Suppl 3):S460-S467. doi: 10.21037/jtd.2017.12.51
43. Gadgeel SM VJ, Kalemkerian GP, et al. Phase II study of maintenance pembrolizumab (pembro) in extensive stage small cell lung cancer (ES-SCLC) patients (pts). J Clin Oncol 2017;35:abstr 8504.

44. Butte MJ, Pena-Cruz V, Kim MJ, et al. Interaction of Human PD-L1 and B7-1. Mol immunol 2008;45:3567-72.

45. Kroemer G, Galluzzi L, Kepp O, et al. Immunogenic cell death in cancer therapy. Annu Rev Immunol 2013;31:51-72.

46. Reits EA, Hodge JW, Herberts CA, et al. Radiation modulates the peptide repertoire, enhances MHC class I expression, and induces successful antitumor immunotherapy. J Exp Med 2006;203:1259-71.

47. Tsuchida T, Yamane H, Ochi N, et al. Cytotoxicity of activated natural killer cells and expression of adhesion molecules in small-cell lung cancer. Anticancer Res 2012;32:887-92.

48. Lee Y, Auh SL, Wang Y, et al. Therapeutic effects of ablative radiation on local tumor require CD8(+) $\mathrm{T}$ cells: changing strategies for cancer treatment. Blood 2009;114:589-95.

49. Lugade AA, Moran JP, Gerber SA, et al. Local Radiation Therapy of B16 Melanoma Tumors Increases the Generation of Tumor Antigen-Specific Effector Cells That Traffic to the Tumor. J Immunol 2005;174:7516-23.

50. Deng L, Liang H, Burnette B, et al. Irradiation and antiPD-L1 treatment synergistically promote antitumor immunity in mice. J Clin Invest 2014;124:687-95.

51. Golden EB, Chhabra A, Chachoua A, et al. Local radiotherapy and granulocyte-macrophage colonystimulating factor to generate abscopal responses in patients with metastatic solid tumours: a proof-of-principle trial. Lancet Oncol 2015;16:795-803.

52. Borghaei H, Paz-Ares L, Horn L, et al. Nivolumab versus Docetaxel in Advanced Nonsquamous Non-Small-Cell Lung Cancer. New Engl J Med 2015;373:1627-39.

53. Garon EB. Pembrolizumab for the treatment of nonsmall-cell lung cancer. N Engl J Med 2015;372:2018-28.

54. Mansfield AS, Murphy SJ, Peikert T, et al. Heterogeneity of programmed cell death-ligand 1 expression in multifocal lung cancer. Clin Cancer Res 2016;22:2177-82.

55. Gibney GT, Weiner LM, Atkins MB. Predictive biomarkers for checkpoint inhibitor-based immunotherapy. Lancet Oncol 2016;17:e542-e51. 